\title{
ADVANCED PHOTON SOURCE LOW-ENERGY UNDULATOR TEST LINE BUNCH COMPRESSOR BEAM POSITION MONITOR SYSTEM*
}

\author{
R. Lill ${ }^{\dagger}$ \\ Advanced Photon Source, Argonne National Laboratory, Argonne IL
}

\section{Abstract}

This paper describes the design and development of a new beam position monitor (BPM) system designed specifically for the Advanced Photon Source Low-Energy Undulator Test Line (LEUTL) bunch compressor. The photocathode gun used for LEUTL presents new challenges for BPM systems. These challenges warranted the development of a new BPM system with a novel surface acoustic wave filter front-end and low-cost monopulse receiver. The system is designed to simultaneously measure single-shot position in both $\mathrm{x}$ and y planes using amplitude-to-phase signal processing topology. A logarithmic amplifier channel measures the beam intensity, which can be used as an indicator of shotto-shot charge fluctuations. The latest experimental results using these BPMs are presented.

\section{INTRODUCTION}

The design and construction of Advanced Photon Source (APS) Low-Energy Undulator Test Line (LEUTL) has created many new design challenges for the diagnostic group. The latest challenge involves supporting the bunch compressor. The primary goal of the bunch compressor is to provide a higher peak current beam to the LEUTL free electron laser [1].

\section{SYSTEM DESIGN OVERVEIW}

The bunch compressor beam position monitor (BPM) system can be divided into four major subsystems as shown in Fig. 1. These subsystems are the BPM detector, receiver front-end, receiver, and data acquisition. The local oscillator and power supply support the receiver front-end and receiver, as shown in Fig. 1.

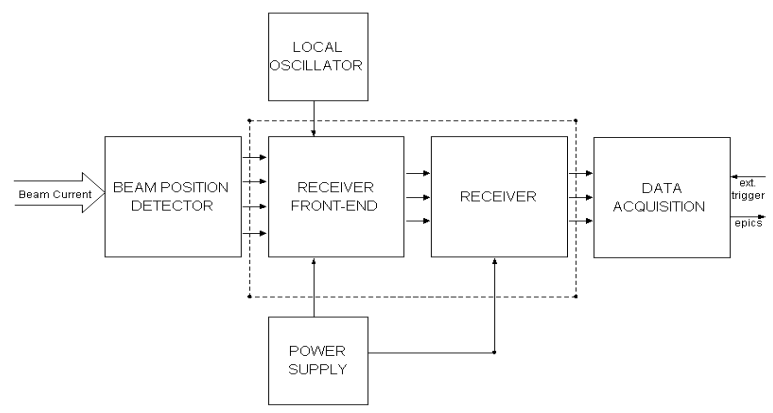

Figure 1: System block diagram.

*Work supported by U.S. Department of Energy, Office of Basic Energy Sciences under Contract No. W-31-109-ENG-38.

†blill@aps.anl.gov

\subsection{Beam Position Detector}

The bunch compressor BPM system employs shorted sband $1 / 4$ wave $(\lambda / 4)$ stripline detectors [2]. The length of the stripline is $28 \mathrm{~mm}$ and the vacuum chamber aperture size is $34 \mathrm{~mm}$. The downstream end of the transmission line is electrically shorted, which also provides the mechanical strength and rigidity to support the stripline pickup. This technique of supporting the stripline results in a transverse electromagnetic wave velocity induced on the stripline that is approximately equal to the beam velocity. The raw stripline output with $0.5 \mathrm{nC}$ from the photocathode gun (PCG) into 50 ohms is shown in Fig. 2.

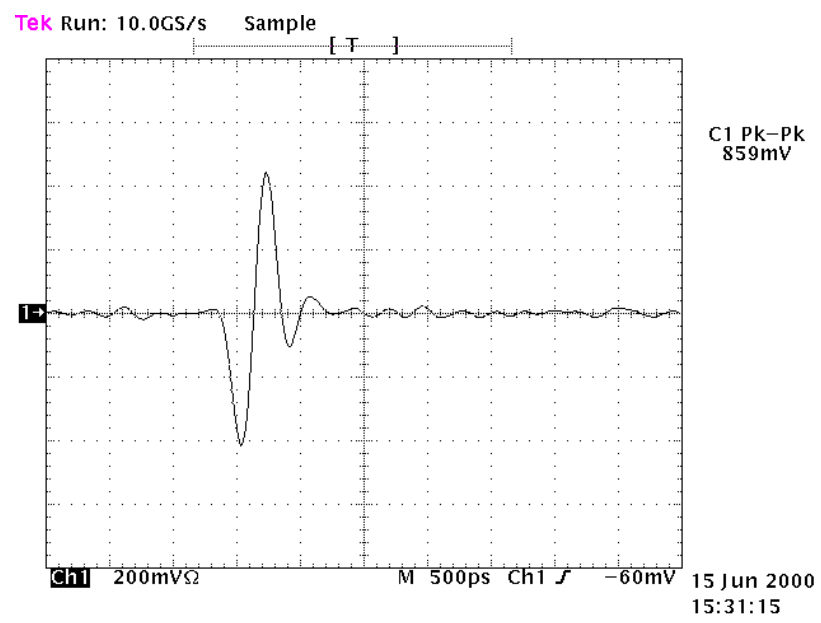

Figure 2: PCG raw stripline output.

\subsection{Receiver Front End}

The receiver front-end, shown in Fig. 3, provides the critical conditioning of a single S-band cycle shown in Fig. 2. The front-end employs very narrow bandwidth $(0.3 \%)$ cavity bandpass resonators to effectively stretch the stripline output. The band pass filters are single-pole 70.7-ohms copper coaxial cavity resonators. The four band pass filters shown in Fig. 3 are matched to less than $0.2 \mathrm{~dB}$ in amplitude and 7 degrees phase in the passband to insure $<100-\mu \mathrm{m}$ offsets. The center frequency (2856 $\mathrm{MHz}$ ) is down-converted to an intermediate frequency of $350 \mathrm{MHz}$. The local oscillator supplies the mixer with +5 $\mathrm{dBm}$ at $3206 \mathrm{MHz}$. The mixer output is at $350 \mathrm{MHz}$ and is matched to a wide band attenuator. The low pass filters provide the filtering for the image and spurious rejection. The intermediate frequency bandwidth is limited to 3.5 $\mathrm{MHz}$ by surface acoustic wave (SAW) filters. The SAW 
filters have matching networks on the input and output that enables us to match the SAW filters in phase and amplitude across the $3.5 \mathrm{MHz}$. The SAW filter is a very economical (\$7/filter) solution because they are a standard product. The signals are then amplified by $26 \mathrm{~dB}$ and fed into the "rat race" hybrid network. The rat race hybrid provides predictable and stable performance over the required bandwidth and temperature range.

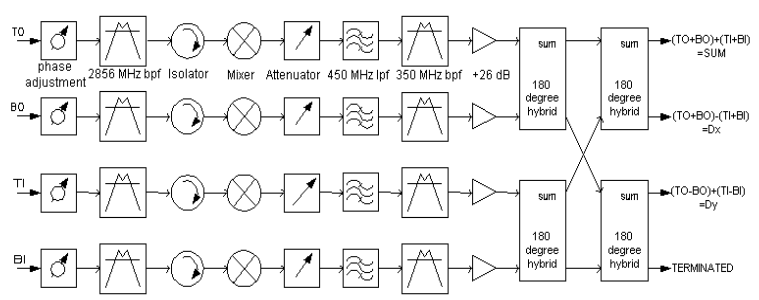

Figure 3: Bunch compressor receiver front-end block diagram.

The stripline output signals, which are proportional to the location of the beam relative to the vacuum chamber, are labeled as top outside (TO), bottom outside (BO), top inside (TI), and bottom inside (BI). The four input signals are paired TO/BO and TI/BI and fed into the first two hybrids, where the sums and differences are derived. The second two hybrids provide as outputs the sum, delta X, and delta $\mathrm{Y}$ of the four input signals. When the beam is centered in the vacuum chamber, the difference signals are nulled and the sum signal is maximum. Movement of the beam off center will result in a decrease in the sum signal along with a corresponding increase in the difference signals. Figure 4 shows the sum and a difference signals after the front-end with a PCG impulse input. The signal envelop shape and duration are set by the intermediate electromagnetic SAW filters.

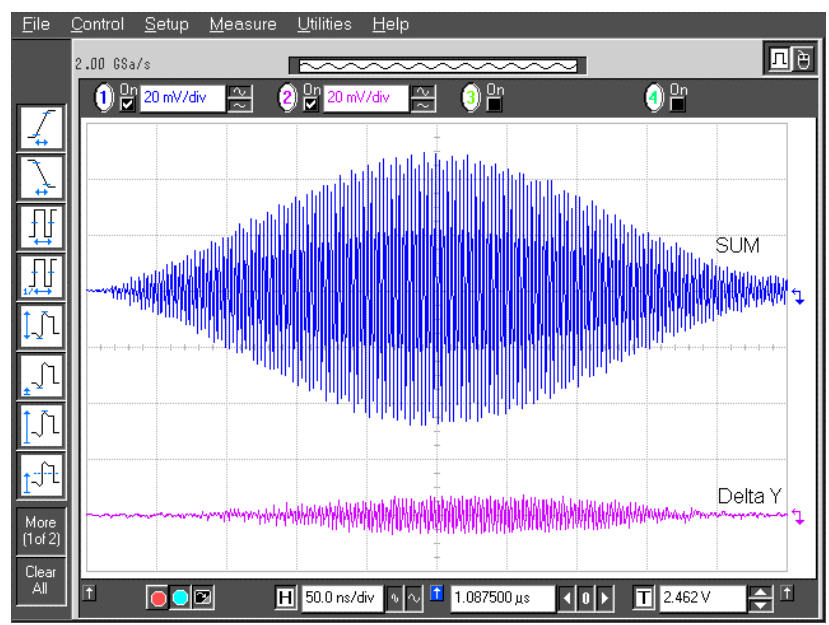

Figure 4: Receiver front-end conditioned output.

\subsection{Receiver}

The receiver topology used is a monopulse amplitudeto-phase (AM/PM) technique for measuring the beam position in the $\mathrm{x}$-and $\mathrm{y}$-axes and a logarithmic amplifier channel for measuring the beam intensity. This receiver was specially designed to measure single-pass beam position for transport line applications. The receiver measures simultaneous horizontal and vertical position data for a single-pass beam. It also has a beam-based trigger, which is not used for this application. The receiver can also run in the commutation mode, where jSum is commutated 0 or 180 degrees in the vector adder section to compensate for phase errors in the limiters over extended dynamic ranges. It was decided this feature would not be necessary to meet design requirements for this application.

Figure 5 is the block diagram of the monopulse receiver. The delta inputs to the receiver are first amplified by $12 \mathrm{~dB}$ in the vector adder section to effectively increase the sensitivity and set the system noise figure. The vector adder then processes the sum and delta signal through 90-degree hybrids so the sum relative to delta is in quadature. The signals $\mathrm{jSum}+\mathrm{dx}$ and jSum+dy are filtered and then fed into the Analog Devices AD $8309 \log /$ limiter. The AD 8309 is the key component to the development of this receiver. In general, the limiter output is an amplified, clipped version of the input waveform that preserves the phase information. The result is a nearly square wave output, which retains the relative phase information of the input waveform. In reality, the limiter output is a by-product of algorithm used to calculate the log of the input signal. The video detector shown is implemented with an exclusive OR-type phase detector with a linear voltage vs. phase output with a sensitivity of $5 \mathrm{~V} / 90$ degrees. The AM/PM transfer function described below is proportional to normalized beam position $(\Delta / \Sigma)$.

$$
\text { Eout }=3.5 \times \tan ^{-1}(4.5 \times \Delta / \Sigma)
$$

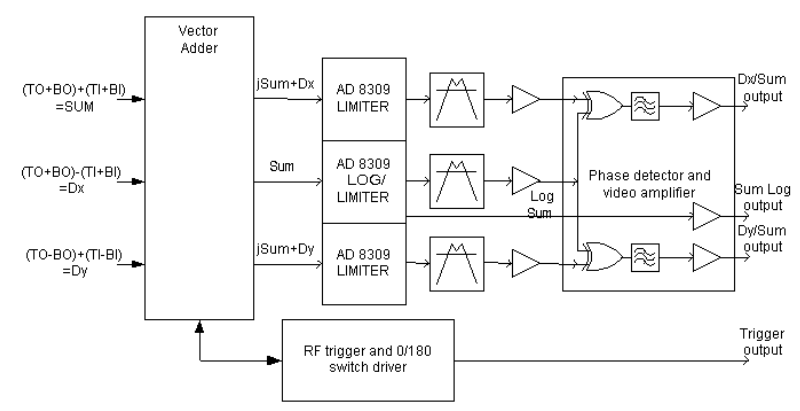

Figure 5: Receiver block diagram. 


\subsection{Data Acquisition}

Data acquisition is accomplished using a VXI-based module, the "envelop detector module model number X018." It was originally designed by Los Alamos National Laboratory and later modified by Argonne National Laboratory. The VXI card has eight input channels, with each having its own sample/hold and 12bit AD574A A/D converter. The 12-bit A/D are bipolar strapped with $\mathrm{a} \pm 5 \mathrm{~V}$ input range.

\section{PERFORMANCE}

We have built and installed four BPMs to support the LEUTL bunch compressor. The BPMs are required to measure bunch lengths as short as 200 fs. The BPM system was designed to meet a $15-\mu \mathrm{m}$ single-shot resolution over a $30-\mathrm{dB}$ dynamic range. The measured resolution can be seen in Fig. 6. The data were taken using the rf gun with an 8-ns macropulse duration. The noise at the high end is dominated by the 12-bit analog-todigital-converter quantization uncertainty. The noise from the receiver and front-end contribute to the power range below $-25 \mathrm{dBm}$. The noise measurement in Fig. 6 was accomplished without the use of receiver commutation circuit, which extends the operating range. The commutation cycle requires two passes of the beam per measurement; for this application it was more important to have true single-shot measurements. The resolution can be improved by selecting a higher resolution analog-todigital converter and implementing the commutation circuit to extend the range.

The sum logarithmic amplifier channel measures the beam intensity, which is used as an indicator of shot-toshot charge fluctuations. The BPM sum channels were calibrated using integrating current transformer monitors upstream and downstream of the BPMs. The additional BPM current monitor outputs aid in tuning and adjusting the bunch compressor. The data obtained from these measurements can also serve as a loss monitor.

The BPMs have also been a useful diagnostic to control the linac accelerating high-power rf. The horizontal position from the BPM at the center of the chicane is used in a feedback loop to effectively hold the energy constant [3].
Typical BPM System Noise Curve

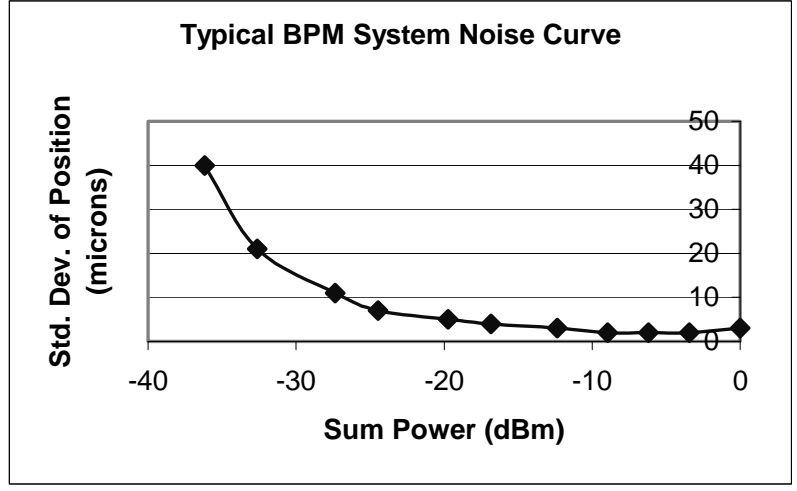

Figure 6: Measured system resolution limit.

\section{DISCUSSION}

We have developed a monopulse-amplitude-to-phasemonopulse BPM system that is suitable for all single-pass transport applications at APS. The design is versatile from the standpoint that the front-end can be reconfigured for other applications because it utilizes discrete components. The receiver was designed with a center operating frequency of $350 \mathrm{MHz}$, which is the APS storage ring rf frequency. The single-shot noise of the receiver without the front-end is about $1 \mu \mathrm{m}$ in a $25-\mathrm{dB}$ dynamic range. This will enable us to use this design in many other applications at APS.

\section{ACKNOWLEDGEMENTS}

The author would like to acknowledge Glenn Decker and Om Singh for many helpful discussions. Robert Keane and Chuck Gold provided valuable electrical measurement and assembly expertise.

\section{REFERENCES}

[1] M. Borland, J. Lewellen, S. Milton "A Highly Flexible Bunch Compressor for the APS LEUTL FEL," Proceedings of the XX International Linac Conference, SLAC-R-561, pp. 863-865 (2001).

[2] A. Gorski, R. Lill, "Construction and Measurement Techniques for the APS LEUTL Project RF Beam Position Monitors," Proceedings of the 1999 Particle Accelerator Conference, New York, pp. 1411-1413 (1999).

[3] M. Borland, J. Lewellen, "Initial Characterization of Coherent Synchrotron Radiation Effects in the Advanced Photon Source Bunch Compressor," these proceedings. 